PROCEEDINGS OF THE

AMERICAN MATHEMATICAL SOCIETY

Volume 136, Number 12, December 2008, Pages 4211-4221

S 0002-9939(08)09576-2

Article electronically published on July 15, 2008

\title{
NEW FORMULAS OF THE BERGMAN KERNELS FOR COMPLEX ELLIPSOIDS IN $\mathbb{C}^{2}$
}

\author{
JONG-DO PARK \\ (Communicated by Mei-Chi Shaw)
}

\begin{abstract}
We compute the explicit formula of the Bergman kernel for a nonhomogeneous domain $\left\{\left(z_{1}, z_{2}\right) \in \mathbb{C}^{2}:\left|z_{1}\right|^{4 / q_{1}}+\left|z_{2}\right|^{4 / q_{2}}<1\right\}$ for any positive integers $q_{1}$ and $q_{2}$. We also prove that among the domains $D_{p}:=$ $\left\{\left(z_{1}, z_{2}\right) \in \mathbb{C}^{2}:\left|z_{1}\right|^{2 p_{1}}+\left|z_{2}\right|^{2 p_{2}}<1\right\}$ in $\mathbb{C}^{2}$ with $p=\left(p_{1}, p_{2}\right) \in \mathbb{N}^{2}$, the Bergman kernel is represented in terms of closed forms if and only if $p=$ $\left(p_{1}, 1\right),\left(1, p_{2}\right)$, or $p=(2,2)$.
\end{abstract}

\section{Introduction and statement of main results}

Let $D$ be a bounded domain in $\mathbb{C}^{n}$. The Bergman space $L_{a}^{2}(D)$ is the space of holomorphic square integrable functions on $D$. For any $z \in D, \Phi_{z}: L_{a}^{2}(D) \rightarrow \mathbb{C}$ defined by $\Phi_{z}(f)=f(z)$ is a linear functional on $L^{2}(D)$ and by the Riesz representation theorem, there is a unique $K_{z}(\cdot) \in L_{a}^{2}(D)$ such that $\Phi_{z}(f)=\left\langle f(\cdot), K_{z}(\cdot)\right\rangle$, namely

$$
f(z)=\int_{D} f(w) \overline{K_{z}(w)} d V(w)
$$

for all $f \in L_{a}^{2}(D)$. Define $K_{D}(z, w)=\overline{K_{z}(w)}$, the Bergman kernel for $D$. The Bergman kernel depends on the choice of $D$ and is also represented by

$$
K_{D}(z, w)=\sum_{\alpha} \phi_{\alpha}(z) \overline{\phi_{\alpha}(w)},
$$

where $\left\{\phi_{\alpha}\right\}_{\alpha}$ is the complete orthonormal basis of the Bergman space $L_{a}^{2}(D)$. The Bergman kernel has been used as one of the main tools in the function theory of several complex variables.

Now we concentrate on computing the explicit formula of the Bergman kernel. If $D$ is a homogeneous domain in $\mathbb{C}^{n}$, then Hua [13] and Yin [17] computed the explicit formula of the Bergman kernel for classical domains and exceptional domains, respectively. Recently many mathematicians [5, 6, 7, 8, 9, 10, 11, 18, have made efforts to find the explicit formulas of the Bergman kernels for nonhomogeneous domains.

Received by the editors February 28, 2007.

2000 Mathematics Subject Classification. Primary 32A25; Secondary 33D70.

Key words and phrases. Bergman kernel, homogeneous domains, hypergeometric function, complex ellipsoids.

The author was supported by Korea Research Foundation Grant 2005-070-C00007 and partially supported by BK21 CoDiMaRO.

(C)2008 American Mathematical Society Reverts to public domain 28 years from publication 
Consider the complex ellipsoids or egg domains $D_{p}:=\left\{z \in \mathbb{C}^{n}: \sum_{j=1}^{n}\left|z_{j}\right|^{2 p_{j}}<\right.$ $1\}$, where $p=\left(p_{1}, \ldots, p_{n}\right)$ for $p_{j}>0$. The precise growth estimate of the Bergman kernel near a boundary point on the complex ellipsoid was studied in [1]. However, it is not easy to get the closed forms of the Bergman kernel for $D_{p}$.

In the case when $p_{1}, \ldots, p_{n}$ are reciprocals of positive integers, Zinov'ev [19] computed the Bergman kernel for $D_{p}$ explicitly. What happens if each $p_{j}$ is a positive integer? The only known case is when $p=\left(1, \ldots, 1, p_{n}\right), p_{n}>0$, for which S. Bergman 4 and J. P. D'Angelo 6, 7] obtained the Bergman kernel.

In this paper we study the Bergman kernel for the complex ellipsoids $D_{p}$ in $\mathbb{C}^{2}$ and compute the explicit formula of the Bergman kernel for $D_{(2,2)}$, which is not homogeneous. For complete Reinhardt domains it is a standard method for computing the Bergman kernel and Szegö kernel to use (1.1), since we can choose $\phi_{\alpha}(z)=z^{\alpha} /\left\|z^{\alpha}\right\|$. This method is called a series method. In this case it is convenient to introduce hypergeometric functions [1, 8]. Let $(a)_{m}=\frac{\Gamma(a+m)}{\Gamma(a)}$, that is, $(a)_{0}=1,(a)_{m}=a(a+1) \cdots(a+m-1)$ for $m=1,2, \ldots$, where $\Gamma$ is the Gamma function. The notation $(a)_{m}$ is called the Pochhammer symbols. For multi-indices $a=\left(a_{1}, \ldots, a_{n}\right)$ and $m=\left(m_{1}, \ldots, m_{n}\right)$, we define $(a)_{m}=\prod_{j=1}^{n}\left(a_{j}\right)_{m_{j}}$. Then one of Appell's multivariate hypergeometric functions is defined by

$$
F_{A}^{(n)}(a ; b ; c ; z):=\sum_{m} \frac{(a)_{|m|}(b)_{m}}{(c)_{m} m !} z^{m}
$$

where $z=\left(z_{1}, \ldots, z_{n}\right) \in \mathbb{C}^{n}, a \in \mathbb{C}, b=\left(b_{1}, \ldots, b_{n}\right), c=\left(c_{1}, \ldots, c_{n}\right)$, and $|m|=\sum_{j=1}^{n} m_{j}$. The series $F_{A}^{(n)}(a ; b ; c ; z)$ converges when $\sum_{j=1}^{n}\left|z_{j}\right|<1$ and diverges when $\sum_{j=1}^{n}\left|z_{j}\right|>1$. This is a multivariate version of the classical EulerGauss hypergeometric function which appears in a theory of the complex plane. That is to say, $F_{A}^{(1)}(a ; b ; c ; z)$ is the classical Euler-Gauss hypergeometric function ${ }_{2} F_{1}(a, b ; c ; z)=F(a, b ; c ; z)$. Explicit formulas of ${ }_{2} F_{1}(a, b ; c ; z)$ are known for specific $a, b, c$, and $z$ (see [12]). If $n=2$, then $F_{A}^{(2)}\left(a ; b_{1}, b_{2} ; c_{1}, c_{2} ; z_{1}, z_{2}\right)$ is

$$
\frac{\Gamma\left(c_{1}\right) \Gamma\left(c_{2}\right)}{\Gamma(a) \Gamma\left(b_{1}\right) \Gamma\left(b_{2}\right)} \sum_{k_{1}=0}^{\infty} \sum_{k_{2}=0}^{\infty} \frac{\Gamma\left(k_{1}+k_{2}+a\right) \Gamma\left(k_{1}+b_{1}\right) \Gamma\left(k_{2}+b_{2}\right)}{\Gamma\left(k_{1}+c_{1}\right) \Gamma\left(k_{2}+c_{2}\right) k_{1} ! k_{2} !} z_{1}^{k_{1}} z_{2}^{k_{2}}
$$

which is usually called $F_{2}\left(a ; b_{1}, b_{2} ; c_{1}, c_{2} ; z_{1}, z_{2}\right)$. This hypergeometric series $F_{2}$ plays an important role in physics and chemistry, and Opps, Saad, and Srivastava 15 obtained some reduction and transformation formulas of $F_{2}$ in the case when the parameters $a, b_{1}, b_{2}, c_{1}$, and $c_{2}$ are positive integers. But we need the case when the parameters $c_{1}$ and $c_{2}$ are specific rational numbers, when we intend to compute the Bergman kernel for $D_{p}$ in $\mathbb{C}^{2}$. In this paper we obtain the Bergman kernel and the Szegó kernel for $D_{(2,2)}=\left\{\left(z_{1}, z_{2}\right) \in \mathbb{C}^{2}:\left|z_{1}\right|^{4}+\left|z_{2}\right|^{4}<1\right\}$ using the formulas of $F_{2}\left(1 ; 1,1, \frac{1}{2}, \frac{1}{2} ; z_{1}, z_{2}\right)$ and $F_{2}\left(2 ; 1,1, \frac{1}{2}, \frac{1}{2} ; z_{1}, z_{2}\right)$, which are obtained in Section 2 and Section 3 . 
Theorem 1.1. The Bergman kernel $K$ and the Szegö kernel $S$ for $D_{(2,2)}=\left\{\left(z_{1}, z_{2}\right)\right.$ $\left.\in \mathbb{C}^{2}:\left|z_{1}\right|^{4}+\left|z_{2}\right|^{4}<1\right\}$ are given by

$$
\begin{aligned}
& K\left(\left(z_{1}, z_{2}\right),\left(w_{1}, w_{2}\right)\right)= \frac{\zeta_{1}\left(\pi+2 \arcsin \zeta_{1}\right) f\left(\zeta_{1}^{2}, \zeta_{2}^{2}\right)}{\pi^{3}\left(1-\zeta_{1}^{2}\right)^{\frac{3}{2}}\left(1-\zeta_{1}^{2}-\zeta_{2}^{2}\right)^{3}}+\frac{\zeta_{2}\left(\pi+2 \arcsin \zeta_{2}\right) f\left(\zeta_{2}^{2}, \zeta_{1}^{2}\right)}{\pi^{3}\left(1-\zeta_{2}^{2}\right)^{\frac{3}{2}}\left(1-\zeta_{1}^{2}-\zeta_{2}^{2}\right)^{3}} \\
&+\frac{8 \zeta_{1} \zeta_{2}}{\pi^{2}\left(1-\zeta_{1}^{2}-\zeta_{2}^{2}\right)^{3}}+\frac{2 g\left(\zeta_{1}^{2}, \zeta_{2}^{2}\right)}{\pi^{3}\left(1-\zeta_{1}^{2}\right)\left(1-\zeta_{2}^{2}\right)\left(1-\zeta_{1}^{2}-\zeta_{2}^{2}\right)^{2}} \\
& S\left(\left(z_{1}, z_{2}\right),\left(w_{1}, w_{2}\right)\right)= \frac{2 \zeta_{1} \zeta_{2}}{\pi^{2}\left(1-\zeta_{1}^{2}-\zeta_{2}^{2}\right)^{2}}+\frac{2}{\pi^{3}\left(1-\zeta_{1}^{2}-\zeta_{2}^{2}\right)} \\
&+\frac{\zeta_{1}\left(1-\zeta_{1}^{2}+\zeta_{2}^{2}\right)\left(\pi+2 \arcsin \zeta_{1}\right)}{\pi^{3}\left(1-\zeta_{1}^{2}-\zeta_{2}^{2}\right)^{2}\left(1-\zeta_{1}^{2}\right)^{\frac{1}{2}}}+\frac{\zeta_{2}\left(1-\zeta_{2}^{2}+\zeta_{1}^{2}\right)\left(\pi+2 \arcsin \zeta_{2}\right)}{\pi^{3}\left(1-\zeta_{1}^{2}-\zeta_{2}^{2}\right)^{2}\left(1-\zeta_{2}^{2}\right)^{\frac{1}{2}}}
\end{aligned}
$$

where $\zeta_{1}=z_{1} \bar{w}_{1}, \zeta_{2}=z_{2} \bar{w}_{2}, f(a, b)=3(1-a)^{2}+6 b(1-a)-b^{2}$, and $g(a, b)=$ $2-a-b-(a-b)^{2}$.

Very recently the author learned that $\mathrm{H}$. Valencourt had also obtained a similar formula as in the above theorem by different methods, but the formula in [16] is more complicated. If we apply Bell's transformation formula 2, 3, of the Bergman kernel under proper holomorphic mappings, we obtain the following corollary.

Corollary 1.2. The Bergman kernel $K$ for $D_{\left(2 / q_{1}, 2 / q_{2}\right)}=\left\{\left(z_{1}, z_{2}\right) \in \mathbb{C}^{2}:\left|z_{1}\right|^{\frac{4}{q_{1}}}+\right.$ $\left.\left|z_{2}\right|^{\frac{4}{q_{2}}}<1\right\}$ for $q_{1}, q_{2} \in \mathbb{N}$ is given by

$$
\begin{aligned}
& K\left(\left(z_{1}, z_{2}\right),\left(w_{1}, w_{2}\right)\right) \\
= & \prod_{i=1}^{2} \frac{1}{q_{i}^{2}\left(z_{1} \bar{w}_{1}\right)^{1-\frac{1}{q_{i}}}} \sum_{j_{1}=1}^{q_{1}} \sum_{j_{2}=1}^{q_{2}} \bar{\omega}_{1}^{j_{1}} \bar{\omega}_{2}^{j_{2}} K_{D_{(2,2)}}\left(\left(z_{1}^{\frac{1}{q_{1}}}, z_{2}^{\frac{1}{q_{2}}}\right),\left(\omega_{1}^{j_{1}} w_{1}^{\frac{1}{q_{1}}}, \omega_{2}^{j_{2}} w_{2}^{\frac{1}{q_{2}}}\right)\right),
\end{aligned}
$$

where $K_{D_{(2,2)}}$ is the Bergman kernel for $D_{(2,2)}$ whose formula is obtained explicitly in Theorem 1.1.

The Bergman kernel is of interest in geometry. For $\alpha, \beta=1,2$ and $z \in D$, we define

$$
g_{\alpha \beta}(z) \equiv \frac{\partial^{2}}{\partial z_{\alpha} \partial \bar{z}_{\beta}} \log K_{D}(z, z) .
$$

The matrix $G_{D}(z) \equiv\left(g_{\alpha \beta}(z)\right)_{\alpha, \beta=1}^{2}$ is called the Bergman metric on $D$. Then the explicit formula of the Bergman kernel enables us to investigate the boundary behavior of the Bergman canonical invariant, which is defined by

$$
J_{D}(z) \equiv \frac{\operatorname{det} G_{D}(z)}{K_{D}(z, z)} .
$$

It is clear that this quantity is invariant under the biholomorphic mappings. From Theorem 1.1 the straightforward but tedious calculation leads to the following formulas. For $(0, w) \in D_{(2,2)}$ we have

$$
G_{D}(0, w)=\left(\begin{array}{cc}
\frac{\pi\left(1+|w|^{2}\right)^{3}\left(3-|w|^{2}\right)}{\sqrt{1-|w|^{4}} H_{1}(w)} & 0 \\
0 & \frac{H_{2}(w)+10|w|^{2} H_{1}(w)^{2}}{\left(1-|w|^{4}\right)^{2} H_{1}(w)^{2}}
\end{array}\right),
$$


where $H_{1}(w)=2 \sqrt{1-|w|^{4}}\left(2+|w|^{4}\right)+3|w|^{2}\left(\pi+2 \arcsin |w|^{2}\right)$ and $H_{2}(w)=$ $72|w|^{2}\left(1-|w|^{4}\right)^{3}+12\left(1-|w|^{4}\right)^{\frac{5}{2}}\left(1+2|w|^{4}\right)\left(\pi+2 \arcsin |w|^{2}\right)$. We also note that

$$
K_{\Omega}(0, w)=\frac{H_{1}(w)}{\pi^{3}\left(1-|w|^{4}\right)^{\frac{5}{2}}} .
$$

Since we have $\lim _{|w| \rightarrow 1} H_{1}(w)=6 \pi$ and $\lim _{|w| \rightarrow 1} H_{2}(w)=0$, we obtain

$$
\lim _{|w| \rightarrow 1} J_{D_{(2,2)}}(0, w)=\frac{40}{9} \pi^{2} .
$$

It is interesting to compare this with Corollary 1 in [14. For the Thullen domain $D_{(2,1)}=\left\{\left(z_{1}, z_{2}\right) \in \mathbb{C}^{2}:\left|z_{1}\right|^{4}+\left|z_{2}\right|^{2}<1\right\}$, the above quantity is also $40 \pi^{2} / 9$. Moreover the boundary limits of $J_{D}$ at strongly pseudoconvex boundary points are the same as $9 \pi^{2} / 2$. See the details in [14.

In Section 4 we express the new formula of the Bergman kernel for $D_{p}$ in $\mathbb{C}^{2}$ in terms of definite integrals. Using Tchebycheff's criterion of specific indefinite integrals, we prove that among the domains $D_{p}$ in $\mathbb{C}^{2}$ with $p=\left(p_{1}, p_{2}\right) \in \mathbb{N}^{2}$, the Bergman kernel is represented as closed forms only if $p=\left(p_{1}, 1\right),\left(1, p_{2}\right)$, or $p=(2,2)$. Thus the domain $D_{(2,2)}$ in Theorem 1.1 is very important in the view of the Bergman kernel.

\section{Hypergeometric functions and Bergman kernel}

Recall that for any nonzero $a, b, c$, the hypergeometric function $F$ in the complex plane is defined by

$$
F(a, b ; c ; z)=\sum_{k=0}^{\infty} \frac{(a)_{k}(b)_{k}}{(c)_{k}(1)_{k}} z^{k}=\frac{\Gamma(c)}{\Gamma(a) \Gamma(b)} \sum_{k=0}^{\infty} \frac{\Gamma(k+a) \Gamma(k+b)}{\Gamma(k+c) \Gamma(k+1)} z^{k} .
$$

In general it is not easy to express the hypergeometric functions in terms of closed forms even in one dimension. There are very few examples such as the following:

$$
F(1,1 ; 1 ; z)=\frac{1}{1-z}, \quad F(1,1 ; 2 ; z)=-\frac{\ln (1-z)}{z} .
$$

In this section we investigate whether each $F(\alpha, 1, \gamma, x)$ for $\alpha, \gamma \in \mathbb{Q}$ can be represented in terms of elementary functions which are not infinite series. The following is our main theorem in this section.

Theorem 2.1. Let $r_{j}=\frac{s_{j}}{p_{j}} \in \mathbb{Q}$ for $j=1,2$.

(i) For $|x|<1$ we have

$$
\begin{aligned}
F\left(r_{1}+r_{2}, 1 ; r_{1} ; x\right) & =\frac{1}{1-x}+\frac{r_{2}}{r_{1}} \frac{x^{1-r_{1}}}{(1-x)^{1+r_{2}}} \int_{0}^{x^{r_{1}}} \frac{d y}{\left(1-y^{\frac{1}{r_{1}}}\right)^{1-r_{2}}} \\
& =\frac{1}{1-x}+\frac{p_{1} r_{2} x^{1-r_{1}}}{(1-x)^{1+r_{2}}} I\left(x^{\frac{1}{p_{1}}}\right),
\end{aligned}
$$

where

$$
I(x)=\int_{0}^{x} \frac{t^{s_{1}-1}}{\left(1-t^{p_{1}}\right)^{1-\frac{s_{2}}{p_{2}}}} d t=\int_{0}^{\left(\frac{x^{p_{1}}}{1-x^{p_{1}}}\right)^{1 / p_{2}}} \frac{y^{\frac{p_{2}}{p_{1}} s_{1}-1}}{\left(1+y^{p_{2}}\right)^{\frac{s_{1}}{p_{1}}+\frac{s_{2}}{p_{2}}}} d y .
$$


(ii) The hypergeometric function $F(\alpha, 1 ; \gamma ; x)$ for $\alpha, \gamma \in \mathbb{Q}$ can be represented in terms of elementary functions which are not infinite series if and only if

$$
\alpha-\gamma \in \mathbb{Z} \quad \text { or } \quad \gamma \in \mathbb{Z} \quad \text { or } \quad \alpha \in \mathbb{Z} .
$$

Now we will prove (i). Note that

$$
F\left(r_{1}+r_{2}, 1 ; r_{1} ; x\right)=1+\sum_{k=1}^{\infty} \frac{\left(r_{1}+r_{2}\right)_{k}}{\left(r_{1}\right)_{k}} x^{k} .
$$

Then we have

$$
\begin{aligned}
g(x) & :=\sum_{k=1}^{\infty} \frac{\left(r_{1}+r_{2}\right)_{k}}{\left(r_{1}\right)_{k}} x^{p_{1} k} \\
& =\frac{r_{1}+r_{2}}{r_{1}} x^{p_{1}}+\frac{\Gamma\left(r_{1}\right)}{\Gamma\left(r_{1}+r_{2}\right)} \sum_{k=2}^{\infty} \frac{\Gamma\left(k+r_{1}+r_{2}\right)}{\Gamma\left(k+r_{1}\right)} x^{p_{1} k} \\
& =\frac{r_{1}+r_{2}}{r_{1}} x^{p_{1}}+\frac{\Gamma\left(r_{1}\right)}{\Gamma\left(r_{1}+r_{2}\right)} \sum_{k=1}^{\infty} \frac{\Gamma\left(k+r_{1}+r_{2}\right)}{\Gamma\left(k+r_{1}\right)} x^{p_{1} k+p_{1}} \\
& +\frac{r_{2} \Gamma\left(r_{1}\right)}{\Gamma\left(r_{1}+r_{2}\right)} \sum_{k=1}^{\infty} \frac{\Gamma\left(k+r_{1}+r_{2}\right)}{\Gamma\left(k+r_{1}+1\right)} x^{p_{1} k+p_{1}} .
\end{aligned}
$$

Since

$$
\int_{0}^{x} t^{s_{1}-1} g(t) d t=\frac{\frac{1}{p_{1}} \Gamma\left(r_{1}\right)}{\Gamma\left(r_{1}+r_{2}\right)} \sum_{k=1}^{\infty} \frac{\Gamma\left(k+r_{1}+r_{2}\right)}{\Gamma\left(k+r_{1}+1\right)} x^{p_{1} k+s_{1}}
$$

we have

$$
g(x)=\frac{r_{1}+r_{2}}{r_{1}} x^{p_{1}}+x^{p_{1}} g(x)+p_{1} r_{2} x^{p_{1}-s_{1}} \int_{0}^{x} t^{s_{1}-1} g(t) d t .
$$

Let $G(x)=\int_{0}^{x} t^{s_{1}-1} g(t) d t$. Then $G^{\prime}(x)=x^{s_{1}-1} g(x)$ and

$$
G^{\prime}(x)-\frac{p_{1} r_{2} x^{p-1}}{1-x^{p_{1}}} G(x)=\frac{r_{1}+r_{2}}{r_{1}} \frac{x^{p_{1}+s_{1}-1}}{1-x^{p_{1}}} .
$$

If we solve this first-order linear differential equation, we have

$$
G(x)=\frac{r_{1}+r_{2}}{r_{1}}\left(1-x^{p_{1}}\right)^{-r_{2}} \int_{0}^{x} \frac{t^{p_{1}+s_{1}-1}}{\left(1-t^{p_{1}}\right)^{1-r_{2}}} d t .
$$

Note that

$$
\int_{0}^{x} \frac{t^{p_{1}+s_{1}-1}}{\left(1-t^{p_{1}}\right)^{1-r_{2}}}=-\frac{1}{p_{1}\left(r_{1}+r_{2}\right)} x^{s_{1}}\left(1-x^{p_{1}}\right)^{r_{2}}+\frac{r_{1}}{r_{1}+r_{2}} \int_{0}^{x} \frac{t^{s_{1}-1}}{\left(1-t^{p_{1}}\right)^{1-r_{2}}} d t .
$$

Thus we have

$$
G(x)=-\frac{1}{s_{1}} x^{s_{1}}+\frac{1}{\left(1-x^{p_{1}}\right)^{r_{2}}} \int_{0}^{x} \frac{t^{s_{1}-1}}{\left(1-t^{p_{1}}\right)^{1-r_{2}}} d t .
$$

Since $F\left(r_{1}+r_{2}, 1 ; r_{1} ; x\right)=1+\left.t^{1-s_{1}} G^{\prime}(t)\right|_{t=x^{\frac{1}{p_{1}}}}$, this completes the proof of (i). To prove (ii) we need the following lemma. 
Lemma 2.2 (Tchebycheff, 1853, 12]). Let $m, n \in \mathbb{Q}$ and $p, q \in \mathbb{Z}$ with $p>0$ and $q \neq 0$. Then the indefinite integral

$$
\int x^{m}\left(a x^{n}+b\right)^{\frac{q}{p}} d x
$$

can be represented in terms of elementary functions if and only if (i) $p=1$, (ii) $\frac{m+1}{n} \in \mathbb{Z}$, (iii) $\frac{m+1}{n}+\frac{q}{p} \in \mathbb{Z}$.

By using Tchebycheff's criterion, the indefinite integral $I(x)$ defined in Theorem 2.1 (i) is represented in terms of elementary functions if and only if $p_{2}=1$, $r_{1} \in \mathbb{Z}, r_{1}+r_{2} \in \mathbb{Z}$. In other words, the hypergeometric function $F(\alpha, 1 ; \gamma, x)$ for nonzero rational numbers $\alpha$ and $\gamma$ can be represented as elementary functions if and only if

$$
\alpha-\gamma \in \mathbb{Z} \quad \text { or } \quad \gamma \in \mathbb{Z} \quad \text { or } \quad \alpha \in \mathbb{Z},
$$

which completes the proof of (ii).

The following example will play an important role in computing the Bergman kernel for $D_{(2,2)}$.

Example 2.3. For any positive integer $n$, we have

$$
\begin{aligned}
F\left(1,1 ; n+\frac{1}{2} ; x\right)=\frac{1-2 n}{1-x}\left\{\sum_{k=1}^{n-1}\right. & \frac{(-1)^{k}}{2 n-2 k-1}\left(\frac{1-x}{x}\right)^{k} \\
& \left.+(-1)^{n}\left(\frac{1-x}{x}\right)^{n-\frac{1}{2}} \arcsin x^{\frac{1}{2}}\right\} .
\end{aligned}
$$

Moreover we have

$$
F\left(1,1 ; \frac{1}{2} ; x\right)=\frac{1}{1-x}+\frac{x^{\frac{1}{2}}}{(1-x)^{\frac{3}{2}}} \arcsin x^{\frac{1}{2}} .
$$

Proof. Let $n \geq 1$. If $p_{1}=p_{2}=2, s_{1}=2 n+1, s_{2}=-2 n+1$, then we have

$$
F\left(1,1 ; n+\frac{1}{2} ; x\right)=\frac{1}{1-x}+\frac{(1-2 n) x^{\frac{1}{2}-n}}{(1-x)^{\frac{3}{2}-n}} I\left(x^{\frac{1}{2}}\right),
$$

where

$$
\begin{aligned}
I(x) & =\int_{0}^{\frac{x}{\left(1-x^{2}\right)^{1 / 2}}} \frac{y^{2 n}}{1+y^{2}} d y \\
& =\int_{0}^{\frac{x}{\left(1-x^{2}\right)^{1 / 2}}}\left[\sum_{k=0}^{n-1}(-1)^{n-k+1} y^{2 k}+\frac{(-1)^{n}}{y^{2}+1}\right] d y \\
& =\sum_{k=0}^{n-1} \frac{(-1)^{n-k+1}}{2 k+1}\left(\frac{x^{2}}{1-x^{2}}\right)^{k+\frac{1}{2}}+(-1)^{n} \arcsin x .
\end{aligned}
$$

Thus we have

$$
\begin{aligned}
F\left(1,1 ; n+\frac{1}{2} ; x\right) & =\frac{1}{1-x}+(1-2 n) \sum_{k=0}^{n-1} \frac{(-1)^{n-k+1}}{2 k+1} \frac{x^{k-n+1}}{(1-x)^{k-n+2}} \\
& +(-1)^{n}(1-2 n) \frac{x^{\frac{1}{2}-n}}{(1-x)^{\frac{3}{2}-n}} \arcsin x^{\frac{1}{2}} .
\end{aligned}
$$


If $n=0$, then $I(x)=\arcsin x$, and we obtain the desired result.

Now we discuss the Bergman kernel for $D_{p}=\left\{z \in \mathbb{C}^{n}: \sum_{j=1}^{n}\left|z_{j}\right|^{2 p_{j}}<1\right\}$. Recall that the monomials $\phi_{\alpha}(z)=\frac{z^{\alpha}}{\left\|z^{\alpha}\right\|_{L^{2}\left(D_{p}\right)}}$ form a complete orthonormal basis for $L_{a}^{2}\left(D_{p}\right)$ in (1.1). The Bergman kernel for $D_{p}$ was obtained in [7, 8, and similarly we can get the Szegö kernel.

Lemma 2.4. Let $p_{j}$ be a positive integer for $j=1, \ldots, n$. Then the Bergman kernel $K(z, w)$ for the domain $D_{p}$ is

$$
\begin{aligned}
K(z, w)= & \frac{\prod_{j=1}^{n} p_{j}}{\pi^{n}} \sum_{\alpha} \frac{\Gamma\left(1+\sum_{j=1}^{n} \frac{\alpha_{j}+1}{p_{j}}\right)}{\prod_{j=1}^{n} \Gamma\left(\frac{\alpha_{j}+1}{p_{j}}\right)}(z \bar{w})^{\alpha} \\
= & \frac{\prod_{j=1}^{n} p_{j}}{\pi^{n}} \sum_{k_{1}=0}^{p_{1}-1} \cdots \sum_{k_{n}=0}^{p_{n}-1}(z \bar{w})^{k} \frac{\Gamma\left(1+\sum_{j=1}^{n} \frac{k_{j}+1}{p_{j}}\right)}{\prod_{j=1}^{n} \Gamma\left(\frac{k_{j}+1}{p_{j}}\right)} \\
& \quad \times F_{A}^{(n)}\left(1+\sum_{j=1}^{n} \frac{k_{j}+1}{p_{j}} ; \mathbf{1} ; \frac{\mathbf{k}+\mathbf{1}}{\mathbf{p}} ;(z \bar{w})^{p}\right)
\end{aligned}
$$

for all $(z, w) \in D_{p} \times D_{p}$. Moreover, the Szegö kernel $S(z, w)$ for the domain $D_{p}$ is

$$
\begin{aligned}
S(z, w)= & \frac{\prod_{j=1}^{n} p_{j}}{2 \pi^{n}} \sum_{\alpha} \frac{\Gamma\left(\sum_{j=1}^{n} \frac{\alpha_{j}+1}{p_{j}}\right)}{\prod_{j=1}^{n} \Gamma\left(\frac{\alpha_{j}+1}{p_{j}}\right)}(z \bar{w})^{\alpha} \\
= & \frac{\prod_{j=1}^{n} p_{j}}{2 \pi^{n}} \sum_{k_{1}=0}^{p_{1}-1} \cdots \sum_{k_{n}=0}^{p_{n}-1}(z \bar{w})^{k} \frac{\Gamma\left(\sum_{j=1}^{n} \frac{k_{j}+1}{p_{j}}\right)}{\prod_{j=1}^{n} \Gamma\left(\frac{k_{j}+1}{p_{j}}\right)} \\
& \times F_{A}^{(n)}\left(\sum_{j=1}^{n} \frac{k_{j}+1}{p_{j}} ; \mathbf{1} ; \frac{\mathbf{k}+\mathbf{1}}{\mathbf{p}} ;(z \bar{w})^{p}\right) .
\end{aligned}
$$

Here we use the notation $\mathbf{1}=1, \ldots, 1$ and $\frac{\mathbf{k}+\mathbf{1}}{\mathbf{p}}=\frac{k_{1}+1}{p_{1}}, \ldots, \frac{k_{n}+1}{p_{n}}$.

In this paper consider $D_{p}=\left\{\left(z_{1}, z_{2}\right) \in \mathbb{C}^{2}:\left|z_{1}\right|^{2 p_{1}}+\left|z_{2}\right|^{2 p_{2}}<1\right\}$ in $\mathbb{C}^{2}$. Then by the preceding lemma, the Bergman kernel is

$$
\sum_{k_{1}=0}^{p_{1}-1} \sum_{k_{2}=0}^{p_{2}-1} c\left(k_{1}, k_{2}\right)(z \bar{w})^{k} F_{2}\left(1+\frac{k_{1}+1}{p_{1}}+\frac{k_{2}+1}{p_{2}} ; 1,1 ; \frac{k_{1}+1}{p_{1}}, \frac{k_{2}+1}{p_{2}} ;(z \bar{w})^{p}\right),
$$

where $c\left(k_{1}, k_{2}\right)=\frac{p_{1} p_{2}}{\pi^{2}} \Gamma\left(1+\sum_{j=1}^{n} \frac{k_{j}+1}{p_{j}}\right) / \prod_{j=1}^{n} \Gamma\left(\frac{k_{j}+1}{p_{j}}\right)$. Thus we need to compute the hypergeometric function $F_{2}\left(1+\gamma_{1}+\gamma_{2} ; 1,1 ; \gamma_{1}, \gamma_{2} ; x, y\right)$. Similarly we also have to compute $F_{2}\left(\gamma_{1}+\gamma_{2} ; 1,1 ; \gamma_{1}, \gamma_{2} ; x, y\right)$ for the Szegö kernel. One can see the following transformation formula in $[8]$.

Lemma 2.5. For any $\gamma_{1}, \gamma_{2}>0$ we have

$$
F_{2}\left(\gamma_{1}+\gamma_{2} ; 1,1 ; \gamma_{1}, \gamma_{2} ; x, y\right)=\frac{\gamma_{1}+\gamma_{2}}{2} \sum_{l=1}^{2} \frac{H_{l}+H_{l}^{*}}{(1-x-y)^{l}}+\frac{1+\frac{1-\gamma_{1}}{\gamma_{1}} x+\frac{1-\gamma_{2}}{\gamma_{2}} y}{(1-x-y)^{2}}
$$

and

$F_{2}\left(1+\gamma_{1}+\gamma_{2} ; 1,1 ; \gamma_{1}, \gamma_{2} ; x, y\right)=\sum_{l=0}^{2} \frac{H_{l}+H_{l}^{*}}{(1-x-y)^{l+1}}+\frac{2}{\gamma_{1}+\gamma_{2}} \frac{1+\frac{1-\gamma_{1}}{\gamma_{1}} x+\frac{1-\gamma_{2}}{\gamma_{2}} y}{(1-x-y)^{3}}$ 
where $H_{l}=c_{l} x^{l} F\left(\gamma_{1}+\gamma_{2}, 1 ; \gamma_{1}+l ; x\right), H_{l}^{*}=c_{l}^{*} y^{l} F\left(\gamma_{1}+\gamma_{2}, 1 ; \gamma_{2}+l ; y\right)$ and

$$
\begin{aligned}
& c_{0}=\frac{\gamma_{2}-1}{\gamma_{1}+\gamma_{2}}, \quad c_{1}=\frac{2\left(\gamma_{2}-1\right)}{\left(\gamma_{1}+\gamma_{2}\right) \gamma_{1}}, c_{2}=\frac{2\left(\gamma_{2}-1\right)}{\left(\gamma_{1}+\gamma_{2}\right) \gamma_{1}\left(\gamma_{1}+1\right)}, \\
& c_{0}^{*}=\frac{\gamma_{1}-1}{\gamma_{1}+\gamma_{2}}, c_{1}^{*}=\frac{2\left(\gamma_{1}-1\right)}{\left(\gamma_{1}+\gamma_{2}\right) \gamma_{2}}, c_{2}^{*}=\frac{2\left(\gamma_{1}-1\right)}{\left(\gamma_{1}+\gamma_{2}\right) \gamma_{2}\left(\gamma_{2}+1\right)} .
\end{aligned}
$$

Remark 2.6. The transformation formula in the above lemma is true for all $\gamma_{1}, \gamma_{2}>$ 0 . But if $\gamma_{1}=1$ or $\gamma_{2}=1$, then the above formula is too complicated to compute. Instead, we can use the following relations:

$$
\begin{aligned}
& F_{2}\left(\alpha ; \beta_{1}, \beta_{2} ; \beta_{1}, \gamma_{2} ; x, y\right)=(1-x)^{-\alpha} F\left(\alpha, \beta_{2} ; \gamma_{2} ; \frac{y}{1-x}\right), \\
& F_{2}\left(\alpha ; \beta_{1}, \beta_{2} ; \gamma_{1}, \beta_{2} ; x, y\right)=(1-y)^{-\alpha} F\left(\alpha, \beta_{1} ; \gamma_{1} ; \frac{x}{1-y}\right) .
\end{aligned}
$$

For example, if $\gamma_{1}=1$, then

$$
F_{2}\left(2+\gamma_{2} ; 1,1 ; 1, \gamma_{2} ; x, y\right)=(1-x)^{-\left(2+\gamma_{2}\right)} F\left(2+\gamma_{2}, 1 ; \gamma_{2}, \frac{y}{1-x}\right),
$$

which is simpler than the above lemma. But Lemma 2.5 is useful when $\gamma_{1}$ and $\gamma_{2}$ are not integers.

\section{Proof of Theorem 1.1}

In this section we prove the main theorem for the Bergman kernel. From Lemma 2.4, the Bergman kernel for $D_{(2,2)}$ is

$$
\begin{aligned}
& \frac{4}{\pi^{2}}\left\{2 \zeta_{1} \zeta_{2} F_{2}\left(3 ; 1,1 ; 1,1 ; \zeta_{1}^{2}, \zeta_{2}^{2}\right)+\frac{3}{4} \zeta_{1} F_{2}\left(\frac{5}{2} ; 1,1 ; 1, \frac{1}{2} ; \zeta_{1}^{2}, \zeta_{2}^{2}\right)\right. \\
& \left.+\frac{3}{4} \zeta_{2} F_{2}\left(\frac{5}{2} ; 1,1 ; \frac{1}{2}, 1 ; \zeta_{1}^{2}, \zeta_{2}^{2}\right)+\frac{1}{\pi} F_{2}\left(2 ; 1,1 ; \frac{1}{2}, \frac{1}{2} ; \zeta_{1}^{2}, \zeta_{2}^{2}\right)\right\},
\end{aligned}
$$

where $\zeta_{j}=z_{j} \bar{w}_{j}$ for $j=1,2$. If $k_{1}=k_{2}=1$, then

$$
F_{2}(3 ; 1,1 ; 1,1 ; x, y)=\frac{1}{(1-x)^{3}} F\left(3,1 ; 1 ; \frac{y}{1-x}\right)=\frac{1}{(1-x-y)^{3}} .
$$

If $k_{1}=1$ and $k_{2}=0$, then using (2.2), we have

$$
F_{2}\left(\frac{5}{2} ; 1,1 ; 1, \frac{1}{2} ; x, y\right)=\frac{1}{(1-x)^{\frac{5}{2}}} F\left(\frac{5}{2}, 1 ; \frac{1}{2} ; \frac{y}{1-x}\right) .
$$

Since we have

$$
F\left(\frac{5}{2}, 1 ; \frac{1}{2} ; \zeta\right)=\sum_{k=0}^{\infty}\left(\frac{4}{3} k^{2}+\frac{8}{3} k+1\right) \zeta^{k}=\frac{3+6 \zeta-\zeta^{2}}{3(1-\zeta)^{3}}
$$

we obtain

$$
F_{2}\left(\frac{5}{2} ; 1,1 ; 1, \frac{1}{2} ; x, y\right)=\frac{f(x, y)}{3(1-x-y)^{3}(1-x)^{\frac{3}{2}}},
$$

where $f(x, y)=3(1-x)^{2}+6(1-x) y-y^{2}$.

If $k_{1}=0$ and $k_{2}=1$, then similarly we have

$$
F_{2}\left(\frac{5}{2} ; 1,1 ; \frac{1}{2}, 1 ; x, y\right)=\frac{f(y, x)}{3(1-x-y)^{3}(1-y)^{\frac{3}{2}}} .
$$


Finally we have to compute $F_{2}\left(2 ; 1,1 ; \frac{1}{2}, \frac{1}{2} ; x, y\right)$. From Example 2.3, we have

$$
\begin{aligned}
& F\left(1,1 ; \frac{1}{2} ; x\right)=\frac{1}{1-x}+\frac{x^{\frac{1}{2}}}{(1-x)^{\frac{3}{2}}} \arcsin x^{\frac{1}{2}}, \\
& F\left(1,1 ; \frac{3}{2} ; x\right)=\frac{\arcsin x^{\frac{1}{2}}}{x^{\frac{1}{2}}(1-x)^{\frac{1}{2}}}, \\
& F\left(1,1 ; \frac{5}{2} ; x\right)=\frac{3}{x}-\frac{3(1-x)^{\frac{1}{2}}}{x^{\frac{3}{2}}} \arcsin x^{\frac{1}{2}}
\end{aligned}
$$

By Lemma 2.5, we have

$$
\begin{aligned}
& F_{2}\left(2 ; 1,1 ; \frac{1}{2}, \frac{1}{2} ; x, y\right) \\
& =\sum_{l=0}^{2} \frac{1}{(1-x-y)^{l+1}}\left\{c_{l} x^{l} F\left(1,1 ; \frac{1}{2}+l, x\right)\right. \\
& \left.\quad+c_{l}^{*} y^{l} F\left(1,1 ; \frac{1}{2}+l, y\right)\right\}+\frac{2(1+x+y)}{(1-x-y)^{3}} \\
& =\frac{f(x, y) x^{\frac{1}{2}} \arcsin x^{\frac{1}{2}}}{2(1-x-y)^{3}(1-x)^{\frac{3}{2}}}+\frac{f(y, x) y^{\frac{1}{2}} \arcsin y^{\frac{1}{2}}}{2(1-x-y)^{3}(1-y)^{\frac{3}{2}}}+\frac{g(x, y)}{2(1-x)(1-y)(1-x-y)^{2}},
\end{aligned}
$$

where $g(x, y)=2-x-y-(x-y)^{2}$. From (3.1) to (3.5), we can obtain the Bergman kernel for $D_{(2,2)}$. Similarly we can also compute the Szegő kernel.

\section{General cases}

So far we have obtained the Bergman kernel for $D_{(2,2)}$. In this section we discuss whether the Bergman kernel for $D_{p}$ with other $p \in \mathbb{N}^{2}$ can be obtained explicitly and prove that the Bergman kernel for $D_{p}$ in $\mathbb{C}^{2}$ when $p \in \mathbb{N}^{2}$ is represented by means of elementary functions if and only if $p=\left(1, p_{2}\right),\left(p_{1}, 1\right),(2,2)$.

If $p \notin \mathbb{N}^{2}$, then there are infinitely many domains $D_{p}$ in $\mathbb{C}^{2}$ whose Bergman kernels are represented by means of elementary functions. For example, using Bell's transformation formula of the Bergman kernel under the proper holomorphic mappings, the Bergman kernel for $D_{\left(1 / q_{1}, 1 / q_{2}\right)}$ for $\left(q_{1}, q_{2}\right) \in \mathbb{N}^{2}$ is obtained as a finite sum in $[8$ and [5].

For any $p_{1}, p_{2} \in \mathbb{N}$, we have to compute

$$
F_{2}\left(1+\frac{k_{1}+1}{p_{1}}+\frac{k_{2}+1}{p_{2}} ; 1,1 ; \frac{k_{1}+1}{p_{1}}, \frac{k_{2}+1}{p_{2}} ; x, y\right),
$$

where $k_{1}=0,1, \ldots, p_{1}-1$ and $k_{2}=0,1, \ldots, p_{2}-1$. Let $\gamma_{1}=\frac{k_{1}+1}{p_{1}}$ and $\gamma_{2}=$ $\frac{k_{2}+1}{p_{2}}$. If we apply Lemma 2.5 , then we have to compute $F\left(\gamma_{1}+\gamma_{2}, 1 ; \gamma_{1}+l ; x\right)$ and $F\left(\gamma_{1}+\gamma_{2}, 1 ; \gamma_{2}+l ; y\right)$ for $l=0,1,2$. Combining Theorem 2.1 (i) and Lemma 2.5, we can obtain the Bergman kernel for $D_{p}$ in $\mathbb{C}^{n}$.

Theorem 4.1. Let $\zeta_{j}=z_{j} \bar{w}_{j}$ for $j=1,2$. 
(i) Then the Bergman kernel for $D_{p}$ in $\mathbb{C}^{2}$ is

$$
\begin{aligned}
K(z, w) & =\sum_{k_{1}=0}^{p_{1}-1} \sum_{k_{2}=0}^{p_{2}-1} c\left(k_{1}, k_{2}\right) \zeta_{1}^{k_{1}} \zeta_{2}^{k_{2}}\left[\sum _ { l = 0 } ^ { 2 } \left\{c_{l}\left(\frac{\zeta_{1}^{p_{1} l}}{1-\zeta_{1}^{p_{1}}}+\frac{p_{1}\left(\gamma_{2}-l\right) \zeta_{1}^{p_{1}\left(1-\gamma_{1}\right)}}{\left(1-\zeta_{1}^{p_{1}}\right)^{1+\gamma_{2}-l}} I_{l}\left(\zeta_{1}\right)\right)\right.\right. \\
& \left.+c_{l}^{*}\left(\frac{\zeta_{2}^{p_{2} l}}{1-\zeta_{2}^{p_{2}}}+\frac{p_{2}\left(\gamma_{1}-l\right) \zeta_{2}^{p_{2}\left(1-\gamma_{2}\right)}}{\left(1-\zeta_{2}^{p_{2}}\right)^{1+\gamma_{1}-l}} I_{l}^{*}\left(\zeta_{2}\right)\right)\right\} \\
& \left.+\frac{c_{3}}{\left(1-\zeta_{1}^{p_{1}}-\zeta_{2}^{p_{2}}\right)^{3}}\left(1+\frac{1-\gamma_{1}}{\gamma_{1}} \zeta_{1}^{p_{1}}+\frac{1-\gamma_{2}}{\gamma_{2}} \zeta_{2}^{p_{2}}\right)\right],
\end{aligned}
$$

where

$$
I_{l}(x)=\int_{0}^{x} \frac{t^{k_{1}+l p_{1}}}{\left(1-t^{p_{1}}\right)^{1-\gamma_{2}+l}} d t, \quad I_{l}^{*}(y)=\int_{0}^{y} \frac{t^{k_{2}+l p_{2}}}{\left(1-t^{p_{2}}\right)^{1-\gamma_{1}+l}} d t .
$$

Here the constants $c_{l}$ and $c_{l}^{*}$ are the same as in Lemma 2.5.

(ii) The Bergman kernel for $D_{p}$ in $\mathbb{C}^{2}$ when $p=\left(p_{1}, p_{2}\right) \in \mathbb{N}^{2}$ is represented by means of elementary functions if and only if $p=\left(1, p_{2}\right),\left(p_{1}, 1\right),(2,2)$.

Proof. By Theorem 2.1 (i), for any $\gamma_{1}, \gamma_{2}>0$,

$$
H_{l}=c_{l} x^{l} F\left(\gamma_{1}+\gamma_{2}, 1, \gamma_{1}+l, x\right)=c_{l} x^{l}\left\{\frac{1}{1-x}+\frac{p_{1}\left(\gamma_{2}-l\right) x^{1-\gamma_{1}-l}}{(1-x)^{1+\gamma_{2}-l}} I\left(x^{\frac{1}{p_{1}}}\right)\right\} .
$$

Similarly we can obtain $H_{l}^{*}$. By Theorem 2.1 (ii) or Tchebycheff's criterion, $H_{l}$ (or $\left.I_{l}(x)\right)$ and $H_{l}^{*}\left(\right.$ or $\left.I_{l}^{*}(y)\right)$ are represented by means of elementary functions if and only if

$$
\gamma_{1} \in \mathbb{Z} \quad \text { or } \quad \gamma_{2} \in \mathbb{Z} \quad \text { or } \quad \gamma_{1}+\gamma_{2} \in \mathbb{Z}
$$

for all $\gamma_{1}=\frac{1}{p_{1}}, \frac{2}{p_{1}}, \ldots, \frac{p_{1}}{p_{1}}$ and $\gamma_{2}=\frac{1}{p_{2}}, \frac{2}{p_{2}}, \ldots, \frac{p_{2}}{p_{2}}$. If $p=\left(1, p_{2}\right),\left(p_{1}, 1\right)$, then condition (4.1) is true trivially. If $p=(2,2)$, then $\gamma_{1}, \gamma_{2} \in\left\{\frac{1}{2}, 1\right\}$ and it is clear that condition (4.1) is true. However, if $p \neq\left(1, p_{2}\right),\left(p_{1}, 1\right),(2,2)$, then there exists a pair $\left(\gamma_{1}, \gamma_{2}\right)$ which does not satisfy condition (4.1). For example, if we take $\gamma_{1}=\frac{1}{p_{1}}$ and $\gamma_{2}=\frac{1}{p_{2}}$, then $\gamma_{1}+\gamma_{2}$ cannot be a positive integer. Thus we prove (ii) of Theorem 4.1 .

\section{ACKNOWLEDGEMENT}

The author would like to express his gratitude to Professor Chong-Kyu Han for helpful suggestions and discussions.

\section{REFERENCES}

1. P. Appell and J. Kampé de Fériet, Fonctions hypergéométriques et hypersphériques, GauthierVillars, Paris, (1926).

2. S. Bell, Proper holomorphic mappings and the Bergman projection, Duke Math. J. 48 (1981), 167-175. MR610182 (82d:32011)

3. S. Bell, The Bergman kernel function and proper holomorphic mappings, Trans. Amer. Math. Soc. 270 (1982), no. 2, 685-691. MR645338 (83i:32033)

4. S. Bergman, Zur Theorie von pseudokonformen Abbildungen, Mat. Sb. (N.S.) 1(43) (1936), no. $1,79-96$

5. H. P. Boas, S. Fu and E. J. Straube, The Bergman kernel function: Explicit formulas and zeroes, Proc. Amer. Math. Soc. 127 (1999), no. 3, 805-811. MR.1469401 (99f:32037)

6. J. P. D'Angelo, A note on the Bergman kernel, Duke Math. J. 45 (1978), 259-265. MR0473231 $(57: 12906)$ 
7. J. P. D'Angelo, An explicit computation of the Bergman kernel function, J. Geom. Anal. 4 (1994), 23-34. MR1274136 (95a:32039)

8. G. Francsics and N. Hanges, The Bergman kernel of complex ovals and multivariable hypergeometric functions, J. Funct. Anal. 142 (1996), 494-510. MR1423042 (97m:32039)

9. K. Fujita, Bergman transformation for analytic functionals on some balls. Microlocal Analysis and Complex Fourier Analysis, 81-98, World Scientific Publisher, River Edge, NJ, 2002. MR2068530 (2005e:32002)

10. K. Fujita, Bergman kernel for the two-dimensional balls, Complex Var. Theory Appl. 49 (2004), no. 3, 215-225. MR2046397(2005b:32004)

11. S. Gong and X. Zheng, The Bergman kernel function of some Reinhardt domains, Trans. Amer. Math. Soc. 348 (1996), no. 5, 1771-1803. MR1329534 (96h:32032)

12. I.S. Gradshteyn and I.M. Ryzhik, Table of integrals, series, and products, translated from the Russian. Translation edited and with a preface by Alan Jeffrey and Daniel Zwillinger. Seventh edition. Elsevier/Academic Press, Amsterdam, 2007. MR2360010

13. L. K. Hua, Harmonic analysis of functions of several complex variables in the classical domain (in Russian), Translations of Math. Monographs, Vol. 6, Amer. Math. Soc., Providence, RI, 1979. MR:598469 (82c:32032)

14. S. G. Krantz and J. Yu, On the Bergman invariant and curvatures of the Bergman metric, Illinois J. Math. 40 (1996), no. 2, 226-244. MR1398092 (97g:32026)

15. S. B. Opps, N. Saad and H. M. Srivastava, Some reduction and transformation formulas for the Appell hypergeometric function $F_{2}$, J. Math. Anal. Appl. 302 (2005), 180-195. MR.2107356 (2005g:33028)

16. H. Valencourt, Projecteurs sur les espaces de fonctions holomorphes: Propriétés et applications, docteur de l'Université de Poitiers, 2002.

17. W. Yin, Two problems on Cartan domains, J. China Univ. Sci. Tech. 16 (1986), no. 2, 130146. MR900957 (88k:32061)

18. E. H. Youssfi, Proper holomorphic liftings and new formulas for the Bergman and Szegö kernels, Studia Math. 152(2) (2002), 161-186. MR1916548 (2003e:32004)

19. B. S. Zinov'ev, Reproducing kernels for multicircular domains of holomorphy (Russian), Sibirsk. Mat. Ž. 15 (1974), 35-48, 236. MR0333230(48:11555)

Department of Mathematical Sciences, Seoul National University, Seoul 151-747, KOREA

Current address: Department of Mathematics, Pohang University of Science and Technology, San 31, Hyoja-dong, Namgu, Pohang, Kyungbuk, 790-784, Korea

E-mail address: jongdopark@gmail.com, jdpark@postech.ac.kr 